\title{
Utility Optimization of Multi-tenant in Divisible Cloud Resource Allocation
}

\author{
Hong Hui \\ Modern Education Technology Center, Shangqiu Medical College, Henan \\ 476100, China \\ E-mail: hong_8204@sina.com
}

\begin{abstract}
In order to solve the competition allocation problem of divisible cloud resource among multi-tenant, an optimal resource allocation strategy based on utility function is proposed in cloud computing. Through defining the evaluation function and the cost function of resource allocation among competing tenants, a utility optimization model is established. The affect of different cost functions on the optimal allocation results is focused, and we have proved that there exists a unique Nash equilibrium solution of resource allocation in our utility model. Experimantal results show that all of the linear cost function and convex cost function can converge to a unique solution, and the linear cost function can bring an optimal system efficiency for a single tenant and maximize the overall social welfare of multi-tenant in the process of social welfare maximization.
\end{abstract}

Keywords: Cloud computing, Nash equilibrium, Utility function, Cost function

\section{Introduction}

As a new computing mode, cloud computing [1,2] mainly focuses on providing a large-scale resource sharing and a comprehensive application services by the manner of the resource pool. As a result, cloud computing can maximize the use of the computing and storage in Internet and achieve an efficient integration of resouces in the whole society. In this process, the management and allocation model of resources is more complex $[3,4,5,6]$, and there are no a universal resource allocation method to deal with all of the application requirements. In view of the similarity with the social economic activities, particularly related to some factors such as the bid, cost (payment) and utility, the economic model is very suitable for solving such problem. Different from the traditional distributed resource allocation and scheduling problem, the objective of this paper is no longer to optimize the execution time of a single task, but is to optimize the utility function and maximize the satisfaction degree of resource allocation in multi-tenant competition environment through mixing the market competition and game mechanism in economics.

In related works, Ref.[7] presents a cloud scheduling model, Cloud Utility Maximizaiton (CUM), which applied approach of Network Utility Maximization into computing resource scheduling. Similar as this paper, CUM does not apply the integer programming model but directed proportion division model. And, a subgradient algorithm for maximizing the utility is designed. But, the utility function in this work only optimizes the execution time not the bidding of users. Ref.[8] presents a cloud game model that can guarantee the Quality of Service (QoS). The model can obtain the optimal resource allocation between tasks and available resources through using Nash equilibrium method. About the utility optimization, for solving the problem of heterogeneity of users' requirements in cloud resource provision, a cloud resource provision strategy based on non-cooperative game utility optimization is proposed in Ref.[9]. But, the optimizing index in [9] is only the execution time of a single task, which could not maximize the 
overall social welfare when the multi-tanant competes for resources. Though our works in this paper use the same resource division model simiar as that in [9], we redefine the utility maximization model that can better reflect the characteristic of competing resource for multi-tenant. In Ref.[10], a grid resource allocation model based on utlity-driven is presented. The problem is that the paper only optimizes the user's bidding and does not consider the cost of competing for resources and the evaluation of resource allocations. The user's bidding, the cost of resource allocation and the evaluation have close relation with each other, especially, the cost will directly affect the value of user's access to resources. In Ref.[11], through defining the evaluation function and cost function to get a new utility function, this utility function has the characteristics of divisible resource allocation, which synchronously takes the bidding, the cost and the evaluation into account. But, the cost is defined as the user's bidding directly, which ignores the influence of the different cost functions.

To solve the above problem, a new utility optimization model based on the multi-tenant is proposed in this paper. In our model, the evaluation function of resource allocation for competing tenants and the cost function are redefined and the optimal express form of the cost function is focused on. Meanwhile, we prove the existence of Nash equilibrium solution of resource allocation. The experimental results show that the linear cost function can lead to the optimal efficiency of the system to maximize the overall social welfare of the multi-tenant.

The rest of the paper is organized as follows. In Section 2 the resource allocation model in cloud computing is described. In Section 3 we prove the existence of Nash equilibrium for utility optimization. In Section 4 the social welfare maximizaiton is applied to solve the cloud resource allocation strategy. In Section 5 we evaluate our strategy by simulation experiments. Finally, in Section 6 we draw conclusion.

\section{Resource Allocation Model}

For $K$ kinds of finite and divisible cloud resource, the bids of $N$ cloud users is $s=\left[s_{1}, s_{2}, \ldots, s_{N}\right]$ and $s_{i}=\left[s_{i}^{1}, s_{i}^{2}, \ldots, s_{i}^{K}\right] . l(s)$ denotes the resource allocation amount and $c(s)$ is the cost function. $l_{i}(s)$ is the amount of resource allocation for user $i, c_{i}(s)$ denotes the cost of user $i$. According to the divisibility of resource and the rule of direct proportion allocation,

$$
l_{i}^{k}(s)=s_{i}^{k} / \sum_{j=1}^{N} s_{j}^{k}
$$

The utility function of user $i$ is defined as the difference between the evaluation function and the paied cost,

$$
U_{i}(s)=w_{i}\left(l_{i}(s)\right)-c_{i}(s, l)
$$

Where $w_{i}(\cdot)$ is the evaluation function of user $i$ for resource allocation and is an increasing function of quadratic differential concave, i.e., $w_{i}^{\prime}\left(l_{i}\right)>0, w^{\prime}{ }_{i}\left(l_{i}\right) \leq 0, l_{i} \in(0,1)$. Then,

$U_{i}^{\prime}(s)=w_{i}^{\prime}\left(l_{i}(s)\right) l_{i}^{\prime}(s)-c_{i}^{\prime}\left(s_{i}\right)$

$U_{i}^{\prime \prime}(s)=w_{i}^{\prime \prime}\left(l_{i}(s)\right)\left[l_{i}^{\prime}(s)\right]^{2}+w_{i}^{\prime}\left(l_{i}(s)\right) l_{i}^{\prime \prime}(s)-c{ }_{i}^{\prime \prime}\left(s_{i}\right)$

Where

$l_{i}^{\prime}(s)=s_{-i} /\left(s_{i}+s_{-i}\right)^{2}>0, l_{i}^{\prime \prime}(s)=-2 s_{-i} /\left(s_{i}+s_{-i}\right)^{3}<0$

If $c{ }^{\prime \prime}{ }_{i}\left(s_{i}\right) \geq 0, \quad U^{\prime \prime}{ }_{i}(s)<0$. The strict concavity of user's utility indicates that user $i$ exsits a unique optimal response for competitors' bids sum $s_{-i}$. For the optimal response to be nonzero, we need the marginal utility when bidding zero to be positive. The happen condistion is

$$
w_{i}^{\prime}(0) / s_{-i}-c_{i}^{\prime}(0)>0 \Rightarrow w_{i}^{\prime}(0) / c_{i}^{\prime}(0)>s_{-i}
$$


The user $i$ 's response $s_{i}$ is defined as the following equation,

$w_{i}^{\prime}\left[s_{i} /\left(s_{i}+s_{-i}\right)\right] \cdot s_{-i} /\left(s_{i}+s_{-i}\right)^{2}-c^{\prime}\left(s_{i}\right)=0$

Let $\theta=\sum_{j} s_{j}$ denote the resource price, and $d(\theta)$ denote the demand function. According to the above model, $s_{i}=d(\theta) \theta$ is the optimal response of $s_{-i}=d(\theta)(1-\theta)$.

\section{Nash Equilibrium}

Based on the above analysis, the convex cost function indicates that users that obtain more resource will pay higher cost. These occur for strictly convex cost function and are classified as discriminatory pricing, while are classified as uniform pricing for the linear cost function such as the proportionally fair allocation. For different cost functions, if games played by users attempt to gain resource, it is important to know whether we can obtain a unique operating point, i.e., a unique Nash equilibrium. The existence of Nash equilibrium can prove that whether the optimization process can converge to the optimal solution. In the follows, we will first discuss the case of the concave cost function $\left(c{ }^{\prime \prime}\left(s_{i}\right)<0\right)$.

According to Equation (1), $w^{\prime}(l)=\theta c^{\prime}(\theta l) /(1-l)=h(l \mid \theta)$. we can get the derivative of $h(l \mid \theta)$ to $\theta, \quad h^{\prime}(l \mid \theta)=\left[(1-l) \theta^{2} c^{\prime \prime}(\theta l)+\theta c^{\prime}(\theta c)\right] /(1-l)^{2}$. Obviously, the sign of $h^{\prime}(l \mid \theta)$ is determined by the quantity $c$ " $(\theta l)+c{ }^{\prime}(\theta l) / \theta(1-l)$. If $c{ }^{\prime \prime}{ }_{i}\left(s_{i}\right)<0$, then $c^{\prime \prime}{ }_{i}\left(s_{i}\right)<-\xi$, for some $\xi>0$ sufficiently small and $s_{1}, s_{2}>0, \quad s \in\left[s_{1}, s_{2}\right]$. Then, $\quad c^{\prime \prime}(\theta l)+c^{\prime}(\theta l) /(\theta(1-l))<-\xi+c^{\prime}(\underline{s}) /\left(\theta-s_{2}\right)<0$ for $\theta$ sufficiently large, where $\underline{s}=\operatorname{argmax}_{s} \in\left[s 1, s_{2}\right] c^{\prime}(s)$. If $\theta>c^{\prime}(\underline{s}) / \xi+s_{2}$, we can know that $f(l \mid \theta)$ is decreasing on $l \in\left[s_{1} / \theta, s_{2} / \theta\right]$. Obviously, there are many evaluation functions $w(\cdot)$ with $\left.w^{\prime \prime}{ }_{i}(\cdot)<0\right)$, where $w^{\prime}(l)$ will intersect $h(l \mid \theta)$ more than once, which implies that there is more than one extremal point. In other words, we can construct a variety of evaluation functions where there are multiple optimal responses. For sufficiently large $\theta$, let $w^{\prime}(l)$ be a decreasing function on the interval $\left[0, s_{1} / \theta\right]$ and $\left[s_{2} / \theta, 1\right]$, where $w^{\prime}\left(s_{1} / \theta\right)=h\left(s_{1} / \theta \mid \theta\right)$ and $w^{\prime}\left(s_{2} / \theta\right)=h\left(s_{2} / \theta \mid \theta\right)$. Then, if $w^{\prime}(l)=h^{\prime}(l \mid \theta)+\delta \sin \left(2 \pi k\left(\theta l-s_{1}\right) /\left(s_{2}-s_{1}\right)\right)$ on $\left[s_{1} / \theta, s_{2} / \theta\right]$ and $\delta$ is sufficiently small to ensure that $w^{\prime}(l)$ is decreasing, we can have an arbitrary number of extremal points by choosing $k$ appropriately. So, the concave cost function can not generate a unique solution.

In the follows, we will discuss that whether $c^{\prime \prime}{ }_{i}\left(s_{i}\right) \geq 0$ can generate a unique Nash equilibrium in the utility model.

Theorem 1 The utility model in this paper can get a unique Nash equilibrium when $c,{ }_{i}\left(s_{i}\right) \geq 0$.

Proof Making $\theta=\sum_{j} s_{j}$ and $l_{i}=s_{i} / \sum_{j} s_{j}$ into Equation (6),

$w_{i}^{\prime}\left(l_{i}\right)\left(1-l_{i}\right)=\theta c_{i}^{\prime}\left(\theta l_{i}\right)$

Every pair $\left(\theta, l_{i}\right)$ that satisfies Equation (7) represents an optimal state for user i. We can interpret these states as demand functions, in which $l_{i}$ is a function of $\theta$. We can get the derivative of Equation (7) to $\theta$,

$$
\begin{aligned}
& {\left[w_{i}^{\prime \prime}\left(l_{i}\right)\left(1-l_{i}\right)-w_{i}^{\prime}\left(l_{i}\right)\right] \partial l_{i} / \partial \theta=c_{i}^{\prime}\left(\theta l_{i}\right)+\theta c_{i}{ }_{i}\left(\theta l_{i}\right)\left[l_{i}+\theta \partial l_{i} / \partial \theta\right]} \\
& \Rightarrow \partial l_{i} / \partial \theta=\frac{\theta l_{i} c_{i}\left(\theta l_{i}\right)+c_{i}^{\prime}\left(\theta l_{i}\right)}{w^{\prime \prime}{ }_{i}\left(l_{i}\right)\left(1-l_{i}\right)-\theta^{2} c^{\prime \prime}\left(\theta l_{i}\right)-w_{i}^{\prime}\left(l_{i}\right)}
\end{aligned}
$$

For $c{ }^{\prime \prime}\left(s_{i}\right) \geqslant 0, \partial l_{i} / \partial \theta<0$. That means that the demand function $d_{i}(\theta)$ which characterize the optimal responses of users are decreasing, where $d_{i}(\theta)=l_{i}(\theta)$ can be obtained from the unique value of $l_{i}$ which solves Equation (7) for a particular $\theta$. Since $d_{i}(0)=1$, the total demand will also be a decreasing function. The Nash equilibrium point is deinfed by total demand being one which occurs at only one level of demand $\theta^{*}$. As a result, there is a unique Nash equilibrium $s_{i}=d_{i}\left(\theta^{*}\right) \theta^{*}$ when $c^{\prime \prime}{ }_{i}\left(s_{i}\right) \geq 0$ in our model.

\section{Social Welfare Maximizaiton}

In economics terms, the efficiency is measured by the the social welfare of all 
participants involved in the allocation process. The social welfare is the sum of all individual utility and the resource provider is also regarded as the game player with the utility $U_{0}(s)=w_{0}\left(l_{0}(s)\right)+\sum_{j \neq 0} c_{j}(s)$. In addition, the social welfare is also can be defined as the sum of the valuations of allocations to all users receiving resource, i.e., $\sum w_{i}\left(x_{i}\right)$. Since we pay more attention to the user behavior influence on the final utility and resource allocation in this paper, the social welfare is the latter. As a result, given a scarce resource, the optimal allocations are obtained from the solution of the following optimazation problem:

$$
\begin{aligned}
& \operatorname{maximize} \sum_{i=1}^{N} w_{i}\left(l_{i}\right) \\
& \text { s.t. } l_{i} \geq 0, \sum_{i=1}^{N} l_{i}=1
\end{aligned}
$$

Thourgh introducing the Lagrange function and the Lagrangian multiplier method,

$L\left(l_{i}\right)=\sum_{i=1}^{N} w_{i}\left(l_{i}\right)+\lambda\left(1-\sum_{i=1}^{N} l_{i}\right)+\sum_{i=1}^{N} \mu_{i} l_{i}$

We can get the first derivative of the Lagrange function to $x_{i}$ and let it be zero, then

$\partial L\left(l_{i}\right) / \partial l_{i}=w_{i}^{\prime}\left(l_{i}\right)-\lambda(0-1)+\mu_{i}=0$

$\Rightarrow w_{i}^{\prime}\left(l_{i}\right)=\lambda-\mu_{i}$

If the user $i$ does not obtain the resource in the optimal allocation outcomes, then

$l_{i}=0 \Rightarrow \mu_{i}=\lambda-w_{i}^{\prime}(0) \geq 0$

Which indicates that the Lagrangian multiplier $\lambda$ must be greater than the margianl valuation $w_{i}^{\prime}(0)$ when the user $i$ does not receive positive allocations.

If the user $i$ receives the resource allocations, i.e., $x_{i}>0$, then the Lagrange function is

$$
L\left(l_{i}\right)=\sum_{i=1}^{N} w_{i}\left(l_{i}\right)+\lambda\left(1-\sum_{i=1}^{N} l_{i}\right)
$$

Similarly, We can get the first derivative of the Lagrange function to $x_{i}$ and let it be zero, then

$\partial L\left(l_{i}\right) / \partial l_{i}=w_{i}^{\prime}\left(l_{i}\right)+\lambda(0-1)=0 \Rightarrow w_{i}^{\prime}\left(l_{i}\right)=\lambda \Rightarrow l_{i}=w_{i}^{\prime-1}(\lambda)$

Which means that the value of the marginal valuations is the Lagrangian multiplier $\lambda$. So, $\lambda$ can be regarded as the value of the margianl valuations of the user group, which determines that which individual member can receive the resource allocations in the population.

In essence, an optimal solution is an allocation of the entire available resource where the marginal valuations for users with positive allocations are all equal. The value of the marginal valuations is the Lagrange multiplier. Users that do not receive positive allocaitons are those highest marginal valuations are less than the value of the Lagrange multiplier. Mathematically, the optimal allocaitons are characterized as follows:

$l_{i}>0 \Leftrightarrow w_{i}^{\prime}\left(l_{i}\right)=\lambda$

$l_{i}=0 \Leftrightarrow w_{i}^{\prime}(0) \leq \lambda$

s.t. $\sum_{i i_{i}>0} l_{i}=\sum_{i l_{i}>0} w_{i}^{\prime-1}(\lambda)=1$

Where $w^{\prime}{ }_{i}^{-1}(\lambda)$ is the inverse function of the $i$-user's valuation function.

The above conclusions indicate that, given an allocation, if user $i$ has a higher marginal valuation than user $j$, the social welfare can be improved by marginally increasing $l_{i}$ and marginally decreasing $l_{j}$. Thus, in an optimal allocation, all active users should have identical marginal valuations.

According to Theory 1, all kinds of cost functions meeting $c{ }^{\prime \prime}\left(s_{i}\right) \geq 0$ can obtain a unique Nash equilibrium, a natural question is how we go about choosing the kind of cost functions. In the next, we consider this question with social welfare maximization as a metric. Since the concave cost function can not get a unique solution, the linear cost functio and the convex cost function will be considered in the follows.

The allocation of user $i$ is determined by Equation (16), $w_{i}^{\prime}\left(l_{i}\right)=\theta c^{\prime}\left(\theta l_{i}\right) /\left(1-l_{i}\right)=h\left(\theta, l_{i}\right)$

$c^{\prime \prime}{ }^{\prime}(\cdot) \geq 0$ implies $h\left(\theta, l_{1}\right)>h\left(\theta, l_{2}\right)$ if $l_{1}>l_{2}$ for all $\theta>0$. If $w^{\prime}(0) \leq h(\theta, 0)$, then user $i$ will not receive a positive allocation at price $\theta$. Otherwise, user $i$ will receive a unique positive 
allocation $l_{i}(\theta)$, which is the solution of $w_{i}^{\prime}\left(l_{i}\right)=h\left(\theta, l_{i}\right)$. And, since $w_{i}^{\prime}(\cdot)$ is a decreasing function of $l_{i}$ and $\theta$ is a strictly increasing function of $l_{i}, l_{i}(\theta)$ is unique. We also have $h\left(\theta_{1}, l\right)>h\left(\theta_{2}, l\right)$ if $\theta_{1}>\theta_{2}$ for all $l \in(0,1)$. This implies that $l_{i}\left(\theta_{1}\right)<l_{i}\left(\theta_{2}\right)$ if $\theta_{1}>\theta_{2}$. As a result, given any cost function $c(s)$, we can find the equilibrium price $\theta$ by solving $\sum_{i} l_{i}(\theta)=1$, from which we can obtain the equlibrium allocations $\left\{l_{i}(\theta)\right\}$.

For a linear cost function $c(s)=k s$, we have $h\left(\theta, l_{i}\right)=\theta k /\left(1-l_{i}\right)$. Though the equilibrium price $\theta$ may differ, every linear cost function yields the same allocations to participating users. Assuming that for $c(s)=k_{1} s$, the equilibrium price is $\theta_{1}$. Then, if $c(s)=k_{2} s, \theta_{2}=\theta_{1} k_{1} / k_{2}$ satisfies all conditions for equlibrium with the same allocations as the case with $c(s)=k_{1} s$. In other words, let $\theta=\theta k$, we can obtain $l_{i}(\theta)$ from $w^{\prime}{ }_{i}\left(l_{i}\right)=\theta /\left(1-l_{i}\right)=h\left(\theta, l_{i}\right)$. The equilibrium allocations for all linear cost functions $\left\{l_{i}\left(\theta^{*}\right)\right\}$ can be expressed in terms of $\theta^{*}$, which is the solution to $\sum_{i} l_{i}(\theta)=1$.

In the next, we will consider a strictly convex cost function $c(s)$. Assuming that for some $\hat{l}$ and $\hat{\theta}$, we have

$$
\hat{\theta} c^{\prime}(\hat{\theta} \hat{l}) /(1-\hat{l})=\hat{\theta}^{*} /(1-\hat{l}) \Rightarrow \hat{\theta} c^{\prime}(\hat{\theta} \hat{l})=\hat{\theta}^{*}
$$

Since $c(s)$ is a strictly convex function, we have $c$ "'( $\cdot)>0$ for $\forall l>\hat{l}$,

$\hat{\theta} c^{\prime}(\hat{\theta} l) /(1-l)>\hat{\theta} c^{\prime}(\hat{\theta} \hat{l}) /(1-l)=\theta^{*} /(1-l)$

For $\forall l<\hat{l}$,

$\hat{\theta} c^{\prime}(\hat{\theta} l) /(1-l)<\hat{\theta} c^{\prime}(\hat{\theta} \hat{l}) /(1-l)=\hat{\theta}^{*} /(1-l)$

It is thus clear that if $h_{c}(\theta, l)=\theta c^{\prime}(\theta l) /(1-l)$ intersects $h(\theta, l)$ at some point $\hat{l}$, then $h_{c}>\grave{h}$ for $\forall l>\hat{l}$ and $h_{c}<h$ for $\forall l<\hat{l}$.

Let $l_{i}^{c}(\theta)$ be obtained from the solution of $w_{i}^{\prime}\left(l_{i}\right)=h_{c}\left(\theta, l_{i}\right)$ and $l_{i}$ be obtained from the solution of $w_{i}^{\prime}\left(l_{i}\right)=h\left(\theta^{*}, l_{i}\right)$. Let $l_{\min }=\min \left\{l_{i}: l_{i}>0\right\}$ be the smallest positive allocation and $l_{\max }=\max \left\{l_{i}\right\}$ be the greatest allocation under a linear cost function. We consider one situation for which $l_{\min }<l_{\max }$, if $\theta_{\min }$ satisfies

$h_{c}\left(\theta_{\text {min }}, l_{\text {min }}\right)=\theta_{\text {min }} c^{\prime}\left(\theta_{\text {min }} l_{\text {min }}\right) /\left(1-l_{\text {min }}\right)=\theta^{*} /\left(1-l_{\text {min }}\right)=h\left(\theta^{*}, l_{\text {min }}\right)$

then, for $\forall l>l_{\min }, h_{c}\left(\theta_{\min }, l\right)>h\left(\hat{\theta}^{*}, l\right)$ and $\sum_{i} l_{i}^{c}\left(\theta_{\min }\right)<\sum_{i} l_{i}=1$.

Similarly, if $\theta_{\max }$ satisfies

$$
h_{c}\left(\theta_{\text {max }}, l_{\max }\right)=\theta_{\text {max }} c^{\prime}\left(\theta_{\text {max }} l_{\text {max }}\right) /\left(1-l_{\text {max }}\right)=\dot{\theta}^{*} /\left(1-l_{\text {max }}\right)=h\left(\dot{\theta}^{*}, l_{\text {max }}\right)
$$

Then, for $\forall l<l_{\max }, h_{c}\left(\theta_{\text {max }}, l\right)<h\left(\theta^{*}, l\right)$ and $\sum_{i} l_{i}^{c}\left(\theta_{\max }\right)>\sum_{i} l_{i}=1$.

The above results imply that the price $\hat{\theta}$ for which $\sum_{i} l_{i}^{c}(\hat{\theta})=1$ must lie strictly in $\left(\theta_{\min }\right.$, $\left.\theta_{\max }\right)$. Furthmore, $h_{c}(\hat{\theta}, l)$ intersects $h\left(\hat{\theta}^{*}, l\right)$ at a point $\hat{l} \in\left(l_{\min }, l_{\max }\right)$.

Then, for $l>\hat{l}$,

$$
h_{c}(\hat{\theta}, l)>h\left(\hat{\theta}^{*}, l\right) \Rightarrow l_{i}>l_{i}^{c}(\hat{\theta})>\hat{l}
$$

For $l<\hat{l}$,

$$
h_{c}(\hat{\theta}, l)<h\left(\theta^{*}, l\right) \Rightarrow l_{i}<l_{i}^{c}(\hat{\theta})<\hat{l}
$$

Let $\triangle E$ is the difference in social welfare between the allocations for a linear cost function $(l)$ and the allocations for a strictly convex cost function $\left(l^{c}(\hat{\theta})\right)$, then

$$
\begin{aligned}
& \Delta E=\sum_{i} w_{i}\left(l_{i}\right)-\sum_{i} w_{i}\left(l_{i}^{c}(\hat{\theta})\right)=\sum_{i} \int_{0}^{l_{i}} w_{i}^{\prime}(l) d l-\sum_{i} \int_{0}^{l_{i}^{c}(\hat{\theta})} w_{i}^{\prime}(l) d l \\
& =\sum_{i: l_{i}>l} \int_{l_{i}^{c}(\hat{\theta})}^{l_{i}^{l}} w_{i}^{\prime}(l) d l-\sum_{i: l_{i}<l} \int_{l_{i}}^{l_{i}^{c}(\hat{\theta})} w_{i}^{\prime}(l) d l
\end{aligned}
$$

Let $l^{+}=\min \left\{l_{i}: \sqcup l_{i}>\hat{l}\right\}, l^{-}=\max \left\{l_{i}: l_{i}<\hat{l}\right\}$, then 


$$
\begin{aligned}
& \Delta E>\sum_{i: l_{i}>l} \int_{l_{i}^{c}(\hat{\theta})}^{l_{i}} \theta^{\square} /\left(1-l^{+}\right) d l-\sum_{i: l_{i}<i} \int_{l_{l_{i}}}^{l_{i}^{c}(\hat{\theta})} \theta^{*} /\left(1-l^{-}\right) d l \\
& =\theta^{*} /\left(1-l^{+}\right) \sum_{i: l_{i}>l}\left[l_{i}-l_{i}^{c}(\hat{\theta})\right]-\theta^{*} /\left(1-l^{-}\right) \sum_{i: l_{i}<l}\left[l_{i}^{c}(\hat{\theta})-l_{i}\right]
\end{aligned}
$$

Since the sum of the resource allocations in the linear cost function and the convex cost function are equal, i.e., one, then

$$
\begin{aligned}
& \sum_{i: l_{i}>l}\left[l_{i}-l_{i}^{c}(\hat{\theta})\right]=\left[\sum_{i: l_{i}>l} l_{i}\right]-\left[\sum_{i} l_{i}\right]+\left[\sum_{i} l_{i}^{c}(\hat{\theta})\right]-\left[\sum_{i: l_{i}>l} l_{i}^{c}(\hat{\theta})\right] \\
& =\sum_{i: l_{i}<l}\left[l_{i}^{c}(\hat{\theta})-l_{i}\right]=\beta>0 \\
& \Rightarrow \Delta E>\beta \theta^{*}\left[1 /\left(1-l^{+}\right)-1 /\left(1-l^{-}\right)\right]>0
\end{aligned}
$$

The above result implies the social welfare is maximized when the cost functions are linear.

It should be pointed out that, this cost function can not optimally maximize social welfare for all users. We consider the case of two users with valuation functions such that $w^{\prime}{ }_{1}(l)>w^{\prime}{ }_{2}(l)$, for all $l \in[0,1]$. We consider the situation with a linear cost function as it yields the optimal efficiency among all cost functions. The allocation fare for user 1 is obtained from the solution to $w^{\prime}{ }_{1}\left(l_{1}\right)=\theta /\left(1-l_{1}\right)$ for some $\theta>0$. This implies that $l_{1}<1$, which in turns implies that $l_{2}>0$. And, their marginal valuation functions must intersect $\theta /(1-l)$, which is an increasing function of $l$. This implies that the users' marginal valuations are not equal at equilibrium unless $l_{1}=l_{2}=1 / 2$, and that can not occur because $w^{\prime}{ }_{1}(1 / 2)>w_{2}{ }_{2}(1 / 2)$. Since both users are active to compete for resource and their marginal valuations are not equal, the social welfare can be improved and thus it is not optimal.

\section{Simulation experiments}

Some simulation experiments are constructed in CloudSim platform [12]. The number of cloud users is set to 10 30. The woakload are randomly generated in the cloud systems. Assuming that the network bandwidth between the tenants and resources can satisfy the transmission requirements of all kinds of tasks. The cost functions are set as: the evaluation function is $w(l)=50 \ln (1+l)$, the linear cost function is $10 s$, the convex cost function is $s^{2}+s$ and the concave cost function is $-s \log (1-s)$. The pseudo-code of the simulation experiemtns are showed in the follows:

/*Multi-tenant Allocation for Cloud Resource Based on Utility*/

1. Input: utility function, evaluation function, cost function, bid

2. Output: resource allocation share, evaluation (social welfare)

3. Initialize parameters of utility function

4. Initialize parameters of multi-tenant

5. Initialize parameters of cloud resrouces

6. For all divisible cloud resources do

7. Return resource capability and estimate the workload

8. End for

9. For each cloud tenant do

10. Send utility function and bid strategy

11. Calculate utility and resource allocation share

12. Save current allocation share and resource price

13. End for

14. If marginal valuations of cloud teants are equal

15. Calculate the solution of equation (2)

16. Save current allocation as optimal solution

17. Else

18. Initialize interations

19. Repeat that until search the solution of $\sum l(\theta)=1$

20. Update interations 
21. Return final allcation solution and total social welfare

Experiment 1 observes the changes of efficiency under different cost functions. It can be seen that from the results of Figure 1, the efficiency of the linear cost function is higher than that of the convex and concave cost function. At the same time, the efficiency of the linear cost function and the convex cost function can achieve stability, while the efficiency of the concave cost function is still a ups and downs in the final stage since it can not generate a unique optimal solution. In contrast, the efficiency of the linear cost function changes more smoothly, the reason is that this function has simpler form, lower computing complexity and can be achieved through the distributed algorithm. The efficiency of the concave cost function intersects with the efficiency of the convex cost function mutually, which indicates that there is no absolute efficiency advantage between them. The difference is the later can generate a unique optimal solution.

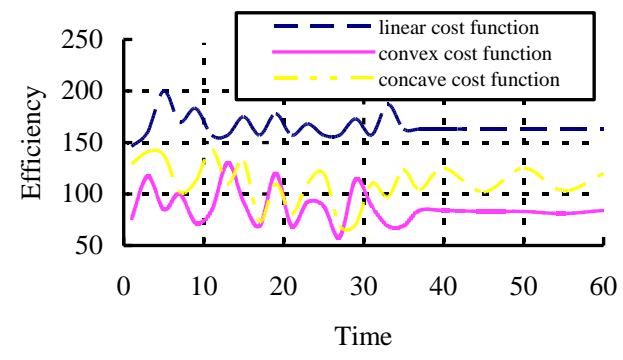

Figure 1. The Efficiency of System

Experiment 2 observes the exsitence of Nash equilibrium. As showed in Figure 2, the stability of resource price is used to verfiy the exsitence of Nash equilibrium. Due to the correspondence relationship between the resource price and resource allocation defined by Equation (2), if we can get a stable resource price, that will indicate the users can get resource allocation maximizing its social welfare. Figure 2 shows that the linear cost function and the convex cost function both can generate the Nash equilibrium, while the concave cost function can not get a unique extreme point since its non-uniqueness optimal response can construct more than one evaluation functions.

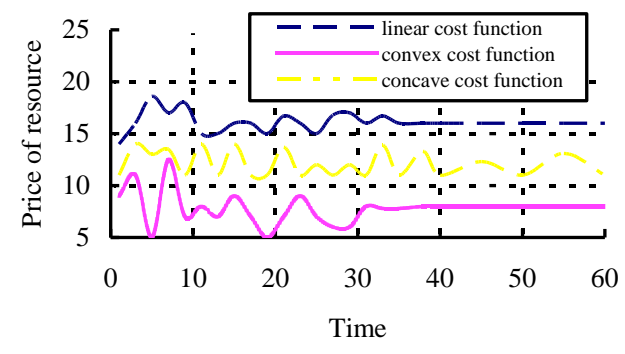

Figure 2. Nash Equilibrium

Experiment 3 observes the changes of social welfare in different numbers of users. Figure 3 indicates that, regardless of what kind of the cost function, the social welfare fluctuates and not has been in a rising trend all the way, which means that no matter kind of cost function all is impossible for us to make the social welfare of all users achieve the maximum at the same time. If the marginal evaluation are not equal, that illustrates the social welfare can be improved further. However, this multi-tenant model will conflict with the resource allocaiton in this situation. The above conclusions further verify that the linear cost function can not make the social welfare of all users achieve the maximum even it can get maximal individual social welfare, in other words, this optimum is a 
mutual optimum each other.

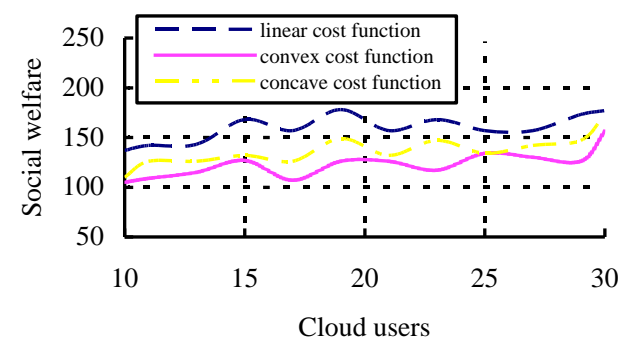

Figure 3. Social Welfare

Experiment 4 observes the resource allocation of coud users. With 10 users as the sample, the cost function is linear. As showed in Figure 4, the resource allocations of users change dynamically. The reason is mainly that the resource share users can obtain depends on the users' bidding. All of the users are trying to maximize their utility in the process of mutual game and competition, so they need to change their bidding strategy constantly until the utility achieves the optimum and gains Nash equilibrium. It can be seen that the resource allocation can converge to the stable state after several rounds of iteration, which indicates that the Nash equilibrium has been reached.

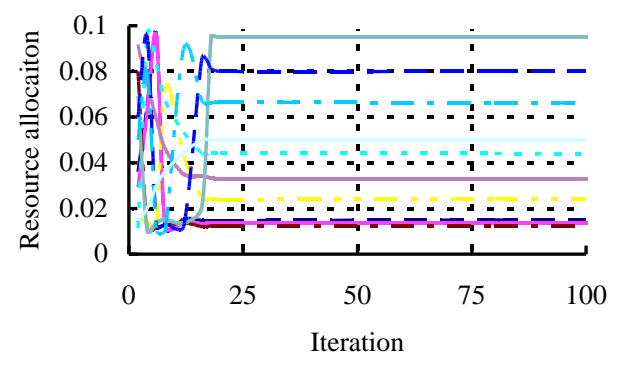

Figure 4. Resource Allocation Share

\section{Conclusion}

A cloud resource allocation strategy for multi-tenant based on utility optimization is presented in this paper. Through establishing the evaluation function and cost function of resource allocation for competing tenants, the utility optimization model is constructed, and the multi-tenant can compete for resources and optimize the utility by the game theory method in economics. At the same time, we focus on how the cost function influences on the resource allocation and prove the exsitence of Nash equilibrium in resource allocation. The experimental results show that the linear cost function will lead to the optimal allocation efficiency and maximize the overall social welfare of the multi-tenant.

\section{References}

[1] R. Buyya, C. S. Yeo and S. Venugopal, "Cloud computing and emerging IT platforms: Vision, hype, and reality for delivering computing as the 5th utility", Future Generation Computer Systems, vol. 25, no. 6, (2009), pp. 599-616.

[2] L. Vaquero, L. Rodero and J. Caceres, "A break in the clouds: towards a cloud definition", SIGCOMM Computer Communication Review, vol. 39, no. 1, (2008), pp. 50-55.

[3] Z. Hai, T. Kun and Z. Xue, "An Approach to Optimized Resource Scheduling Algorithm for Open-Source Cloud Systems", Fifth Annual ChinaGrid Conference, (2010).

[4] B. Xu, C. Zhao and E. Hu, "Job scheduling algorithm based on Berger model in cloud environment", Advances in Engineering Software, vol. 42, no. 7, (2011), pp. 419-425.

[5] M. Mezmaz, N. Melab and Y. Kessaci, "A parallel bi-objective hybrid metaheuristic for energy-aware 
scheduling for cloud computing systems", Journal of Parallel and Distributed Computing, vol. 71, no. 11, (2011), pp. 1497-1508.

[6] S. K. Garg, C. S. Yeo and A. Anandasivam, "Environment-conscious scheduling of HPC applications on distributed Cloud-oriented data centers", Journal of Parallel Distributed Computing, vol. 71, no. 6, (2011), pp. 732-749.

[7] X.-L. Shi and X. Ke, "Utility maximization model of virtual machine scheduling in cloud environment", Chinese Journal of Computers, vol. 36, no. 2, (2013), pp. 252-262.

[8] W. Guiyi, A. V. Vasilakos and X. Naixue, "Scheduling Parallel Cloud Computing Services: An Evolutional Game", 1st International Conference on Information Science and Engineering, (2009).

[9] X. Zhang, Z.-T. He and C.-L. Li, "Cloud resource provision strategy based on non-cooperative game utility optimization", Computer Engineering, vol. 39, no. 7, (2013), pp. 59-62.

[10] Z. Xiao, L. Chun and Q. Qiong, "Utility-driven non-cooperative game model of optimal resource allocation for economic grid", Journal of Computational Information Systems, vol. 7, no. 1, (2011), pp. 190-197.

[11] X. Zhan and Q. Yue, "Cloud resource game allocation based on cooperation", Computer Applications, vol. 34, no. 7, (2014), pp. 1848-1851.

[12] R. N. Calheiros, R. Ranjan and A. Beloglazov, "CloudSim: a toolkit for modeling and simulation of cloud computing environments and evaluation of resource provisioning algorithms", Software-Practice \& Experience, vol. 41, no. 1, (2011), pp. 23-50. 
International Journal of Grid and Distributed Computing

Vol. 10, No. 2 (2017) 